\title{
Biological Risk Factors and Early Developmental Delay Assessment in Infants Using Ages and Stages Questionnaire, Version 3 (ASQ-3)
}

\author{
Sumandeep Kaur ${ }^{1}$, Navdeep S Sidhu', Rajwant Kaur Randhawa ${ }^{3}$ \\ ${ }^{1} \mathrm{PhD}$ Scholar, Desh Bhagat University, Mandi Gobindgarh, Tutor, University College of Nursing, Faridkot, \\ Punjab, India \\ ${ }^{2}$ Assistant Professor, Guru Gobind Singh Medical College, Faridkot, Punjab, India \\ ${ }^{3}$ Director \& Principal, Desh Bhagat Institute of Nursing, Mandi Gobindgarh, Punjab, India \\ Corresponding Author: Navdeep S Sidhu
}

\begin{abstract}
Background: Development during early years of life is very crucial in the context of overall development of an individual. This is especially true for infants who have biological risk factors for maldevelopment. This study aimed to find the association of biological risk factors and developmental delay in infants aged 3-18 months.
\end{abstract}

Materials and Methods: For this study, enrolment of 460 infants was done in the age group of 3-18 months who were attending Immunization Clinic in district level Civil Hospital, Faridkot, Punjab. Developmental assessment in the five areas including Gross motor, Fine motor, Communication, Problem solving and Personal-social development was done using a standardized tool called Ages and Stages Questionnaire, version 3 (ASQ-3).

Results: Most of the study subjects viz., 426 (92.6\%) were born full term whereas 34 (7.3\%) infants were born preterm. Mean birth weight of infants was $2.9 \pm 0.387 \mathrm{kgs}$. Out of the total, $259(56.3 \%)$ study subjects were males and 201(43.7\%) were females. Biological risk factors like gestation age and birth weight had statistically significant association with all domains of developmental delay except in the area of personal social development. On the other hand, gender had no association with any domain of developmental delay.

Conclusion: Gestation age and birth weight are the typical risk factors having significant impact on all domains of developmental delay except for the personal social delay. However, gender of infant had no association with developmental delay.

Keywords: Infant, Gestation age, birth weight, Developmental delay, ASQ

\section{INTRODUCTION}

Advancements in the medical sciences have greatly improved the survival rate of high- risk newborns. This has raised an important issue related to the future development of these children. ${ }^{[1,2]}$ Developmental delays are expected to occur more frequently in these high-risk infants resulting in delay in the achievement of milestones at the expected age. ${ }^{[3]}$ The main causes responsible for this delay are not always clear, but most of these infants have history of some risk factors during intrauterine life or after birth. Most common factors contributing to developmental delay and infant death are prematurity, low birth weight and perinatal maternal complications. ${ }^{[4]}$ According to Piek et al., the gestational age is the predominant factor affecting fine motor skills at school age. ${ }^{[5]}$ Hediger et al. reported that low levels of parental education, younger age of the mother, high gravidity, low birth weight, and preterm labor are significantly associated with delayed motor and social development. $^{[6]}$ Due to the numerous problems associated with having a child afflicted by developmental delay, early 
diagnosis and timely referral are very important and can benefit children with developmental disabilities and their families. ${ }^{[7]}$

Globally, about 7.9 million children are born annually with a serious birth defect of genetic or partially genetic origin which accounts for 6 percent of the total births. Serious birth defects can be fatal at times. For those who do not receive specific and timely intervention and yet survive, these disorders can cause irreversible life-long mental, physical, auditory or visual disability. Special Newborn Care Units (SNCU) Technical Reports have reported that approximate 20 percent of babies discharged from health facilities are found to suffer from developmental delays or disabilities at a later age. ${ }^{[8]}$ Early detection and intervention can bring a change in this trend by reducing the burden of development delay and disability.

\section{MATERIALS AND METHODS}

The present research study was conducted in the immunization setting of the Civil Hospital, Faridkot, Punjab, India which is a district-level public hospital. Enrolment of a total of 460 study subjects who were attending immunization clinic of Civil Hospital, Faridkot was done in the age group of 3-18 months using purposive sampling technique. The detailed methodology of our research study has already been published previously. ${ }^{[9]}$ Sample size was calculated using the power analysis. For participation in the study, written informed consent was taken from the parents of the infants. After the parents' consent 460 infants in age group of 3-18 months were assessed for gross motor, fine motor, communication, problem solving and personal social development using Ages and Stages Questionnaire, version 3 (ASQ-3) tool. Infants with history of major neurological and musculoskeletal injuries were excluded from the study. ASQ-3 is a standard parent reported and user-friendly assessment tool that can be used by primary healthcare providers and caregivers easily.
Techniques used by the researcher to complete the developmental assessment included interview, observation and examination. Content validity of the tool was checked using checklists by a total of 11 experts having sound knowledge of the subject. Five-point Likert's rating scale was used to calculate content validity index (CVI). The CVI for the total instrument was proportion of items rated either 4 or 5 . Calculated CVI was 0.85 for ASQ-3. Reliability of the tool was calculated before use in the present study. This was, $r=0.70$ which is an acceptable level of reliability coefficient.

\section{Statistical methods:}

Data were collected using the structured tool (ASQ-3) and data were entered in the SPSS sheet (SPSS software, version 23, SPSS Inc. Chicago) in an organized form. As per the objectives of study, data were analyzed using descriptive and inferential statistical techniques. Results of study are presented in form of tables and figures. Baseline profile of study subjects was described in frequency and percentages. To find out association between biological risk factors and development delay chi square test was applied. The level of significance was $5 \%$ for this study. The pvalue of $\leq .05$ was considered statistically significant.

\section{RESULTS}

Table 1: Distribution of study subjects as per baseline biological profile, $N=460$

\begin{tabular}{|l|l|l|}
\hline Variable & Frequency & Percentage \\
\hline Gestation & & \\
\hline Term infants & 426 & $92.6 \%$ \\
\hline Preterm infants & 34 & $7.3 \%$ \\
\hline Gender & & \\
\hline Male & 259 & $56.3 \%$ \\
\hline Female & 201 & $43.7 \%$ \\
\hline Birth weight & & \\
\hline Normal birth weight & 429 & $93.3 \%$ \\
\hline Low birth weight & 31 & $6.7 \%$ \\
\hline
\end{tabular}

A total of 460 infants of 3-18 months of age were enrolled in this study. Table 1 depicts the baseline characteristics of the study participants including gestation age, gender and birth weight. Most of the 
study subjects viz., $426(92.6 \%)$ were born at term gestation whereas $34(7.3 \%)$ were born preterm. Majority of subjects had mean birth weight $2.9 \pm 0.387 \mathrm{kgs}$. In addition, out of total 460 subjects $259(56.3 \%)$ were male and 201(43.7\%) were female.
The association of development delay with gestation age has been presented in table 2. All the domains of development were significantly associated with gestation except personal social domain which is not influenced by gestation age.

Table 2: Association of developmental delays with gestation age

\begin{tabular}{|l|l|l|l|l|}
\hline \multirow{2}{*}{ Developmental delay } & Gestation age & Chi square and Df & P value \\
\cline { 2 - 5 } & Term(N=426), frequency $(\%)$ & Preterm(N=34), frequency $(\%)$ & & \\
\hline Communication delay & $19(4.5 \%)$ & $9(26.5 \%)$ & $26.685, \mathrm{Df}=1$ & 0.000 \\
\hline Gross motor delay & $33(7.7 \%)$ & $11(32.7 \%)$ & $22.039, \mathrm{Df}=1$ & 0.000 \\
\hline Fine motor delay & $24(5.6 \%)$ & $15(44.1 \%)$ & $60.098, \mathrm{Df}=1$ & 0.000 \\
\hline Problem solving delay & $34(8 \%)$ & $8(23.5 \%)$ & $9.174, \mathrm{Df}=1$ & 0.007 \\
\hline Personal social delay & $29(6.8 \%)$ & $4(11.8 \%)$ & $1.162, \mathrm{Df}=1$ & 0.219 \\
\hline
\end{tabular}

The association of delay in various domains of developmental with birth weight of infants has been shown in table 3 . It has been observed that developmental delay in various domains is significantly associated with birth weight except the personal social domain. It shows that preterm infants and low birth infants were more prone to developmental delays.

Table 3: Association of developmental delays with birth weight:

\begin{tabular}{|l|l|l|l|l|}
\hline \multirow{2}{*}{ Developmental delay } & \multicolumn{2}{|l|}{ Birth weight } & Chi square and Df & P value \\
\cline { 2 - 6 } & Normal(N=429), frequency (\%) & Low birth weight $(\mathbf{N}=\mathbf{3 1})$, frequency (\%) & & \\
\hline Communication delay & $22(5.1 \%)$ & $6(19.4 \%)$ & $10.236, \mathrm{Df}=1$ & 0.007 \\
\hline Gross motor delay & $34(7.9 \%)$ & $10(32.3 \%)$ & $19.788, \mathrm{Df}=1$ & 0.000 \\
\hline Fine motor delay & $26(6.1 \%)$ & $13(41.9 \%)$ & $47.952, \mathrm{Df}=1$ & 0.000 \\
\hline Problem solving delay & $34(7.95 \%)$ & $8(25.8 \%)$ & $11.141, \mathrm{Df}=1$ & 0.004 \\
\hline Personal social delay & $28(6.5 \%)$ & $5(16.1 \%)$ & $4.003, \mathrm{Df}=1$ & 0.061 \\
\hline
\end{tabular}

In our study, we did not find any significant association of developmental delay with the gender of the infant (table 4). This was true for all the domains of the development which were studied in the present study.

Table 4: Association of developmental delays with gender of infant:

\begin{tabular}{|l|l|l|l|l|}
\hline \multirow{2}{*}{ Developmental delay } & Gender & Chi square and Df & P value \\
\cline { 2 - 5 } & Male(N=259), frequency (\%) & Female(N=201), frequency (\%) & & \\
\hline Communication delay & $16(6.2 \%)$ & $12(6 \%)$ & $0.009, \mathrm{Df}=1$ & 0.544 \\
\hline Gross motor delay & $26(10 \%)$ & $18(9 \%)$ & $0.154, \mathrm{Df}=1$ & 0.410 \\
\hline Fine motor delay & $23(8.9 \%)$ & $16(8 \%)$ & $0.123, \mathrm{Df}=1$ & 0.430 \\
\hline Problem solving delay & $24(9.3 \%)$ & $18(9 \%)$ & $0.013, \mathrm{Df}=1$ & 0.521 \\
\hline Personal social delay & $19(7.3 \%)$ & $14(7 \%)$ & $0.023, \mathrm{Df}=1$ & 0.541 \\
\hline
\end{tabular}

\section{DISCUSSION}

Initial five years of life, commonly referred to as the early childhood is of paramount importance in the developmental history of an individual, as it is a period of life in which basic skills related to motor, cognitive, language and social domains are acquired. This period of life is the time of the fastest growth and is also the phase in which the developing brain and nervous system is most sensitive to nurturing and stimulation. This phase of human development is considered as the foundation for subsequent attainment of educational and vocational goals at the individual's level, and overall human resource and economic development at the population level. ${ }^{[10]}$

Various factors affecting the early childhood development are broadly categorized into biological factors and the environmental factors. In the present study we sought to determine the association between biological risk factors and developmental delays in infants aged 3 to 18 months. The biological risk factors explored in the current study included birth weight, gestation age and gender.

In our study, we found a significant and positive association between the various 
domains of childhood development and birth weight. Similarly, Datar et al. in their study involving the developmental assessment of twins, found that a very low birth weight (less than 1500gm) and moderately low birth weight (1500 to 2499 gm) had pronounced negative effects on parameters of motor and mental development at 9 months and 2 years of age. ${ }^{[11]}$ Whitaker et al conducted a study of nondisabled low birth weight (less than 2000 gm) cohort and followed up 474 nondisabled low birth weight (LBW) infants into their adolescence and studied the occurrence of motor problems and values of intelligence quotient (IQ) score. They found that as compared to normative sample, the non-disabled LBW children had more motor problems and the IQ scores of these LBW children, although were within the normal range but were significantly lower than the population norms.

In a study from Iran which is a developing country like India Tavasoli et al. studied 88 infants including 58 moderately LBW (MLBW) and 30 normal birth weight infants by assessment of motor development using Peabody motor assessment 2 (PDMS2). It was found that motor assessment at $18 \pm 2$ months revealed that there was no statistically significant difference in the gross motor quotient but the MLBW infants had significantly lower fine motor and total motor scores. ${ }^{[13]}$

In a recent study published by Drozd- Dabrowsk et al in 2018, the authors evaluated various risk factors in infancy which were associated with developmental delay in childhood. The results of this study indicated that the most common risk factors in infancy which were more associated with future developmental delay in childhood were: Caesarean section, infections, chronic diseases during pregnancy. In this study it was found that infants with birth weight of less than $2500 \mathrm{gm}$ were three times more likely to develop developmental delay as compare to infants with normal birth weight. ${ }^{\mid}$
In another recent study by Pirhadi et al from Iran, the authors studied the relation of birth weight to the occurrence of developmental delay in children aged 4 to 16 months. In the study it was found that LBW of the child was associated with developmental delay in domains of fine motor movements, problem solving and total scores as assessed by ASQ.

Gestation age is another typical risk factor for developmental delay. In the current study, it was found the gestation age has a significant and positive association with the childhood development.

A study by Kerstjens et al, assessed the effect of gestational age on occurrence of developmental delay in various domains by ASQ among children aged around 4 years. The results of the study showed that the prevalence rate of abnormal scores on the ASQ total-problem scale increased with decreasing gestational age: form $4.2 \%$ among the term born children to $37.5 \%$ among children born at 24-25 weeks of gestation $(p<0.000)$. The risk of abnormal ASQ total score increase exponentially with decreasing gestational age compared with children born at term. ${ }^{[16]}$

A study by Schonhut et al. from Chile in 2015 of 1667 infants, evaluated the association between the gestation age at birth and risk of developmental delay using ASQ at 8 and 18 months of corrected postnatal age. This study found that compared with full term born infants the odds ratio for developmental risk ratio was 1.6 for those born early term, 2.58 for infants born late preterm and 3.01 for those born moderately preterm. An inverse doseresponse relationship between gestational age and risk of developmental delay was found in the study population. ${ }^{[17]}$

In the recently published large Upstate KIDS study by Hochstedler et al from U.S.A, developmental delays were assessed in 5,868 children at 7 time points $(4,8,12,18,24,30$ and 36 months) using ASQ. The authors concluded that the gestation age was inversely associated with 
developmental delays for all gestational ages. $^{[18]}$

In our study we did not find any significant association between gender and developmental delay. Our results are in contrast to the results of study by Soleimani et al, who in their study of 250 infants found that male gender variable had a significant relationship with developmental delay in early childhood. ${ }^{[19]}$ Similarly, our findings are in contrast to few other studies which have found significant relation between the male gender and the occurrence of developmental delays. ${ }^{[20-22]}$

\section{CONCLUSION}

Among the biological risk factors, the gestation age and birth weight have significant impact on various domains of developmental delay except for the personal social domain. However, gender of the infant had no association with childhood developmental delay.

\section{Acknowledgement: None}

\section{Conflict of Interest: None}

\section{Source of Funding: None}

\section{Ethical Approval: Approved}

\section{REFERENCES}

1. Chaman R, Holakouie NK, Golestan B, et al. Neonatal mortality risk factors in a rural part of Iran: a nested case control study. Iranian J Publ Health. 2009; 38:48-52.

2. Soleimani F, Vameghi R, Dadkhah A. Highrisk infants referred to health-care centers in North and East of Tehran and risk factors of motor developmental delay. Hakim Res J. 2009; 12:11-18.

3. Baker RC. Pediatric primary care well-child care. Philadelphia, PA: Lippincott Williams and Wilkins Publishers; 2001.

4. Sajedy F, Alizadeh V. The incidence of motor developmental delay in high-risk infants and effective risk factors in developing of it. Q J Rehabil. 2008; 5:7-12.

5. Piek JP, Dawson L, Smith LM, et al. The role of early fine and gross motor development on later motor and cognitive ability. Hum Movement Sci. 2008; 27:66881.

6. Hediger ML, Overpeck MD, Ruan WJ, et al. Birth weight and gestational age effects on motor and social development. Paediatr Perinat Epidemiol. 2002; 16:33-46.

7. Lin JD, Yen $\mathrm{CF}, \mathrm{Wu} \mathrm{JL}$, et al. The administrative population report on children with developmental delays in Taiwan, 2003 through 2007. Res Dev Disabil. 2009; 30:353-8.

8. Ministry of Health and Family Welfare Government of India. Rashtriya Bal Swasthya Karyakram (RBSK) Child Health Screening and Early Intervention Services under NRHM. Operational Guidelines; 2013.

http://cghealth.nic.in/nhmcg/Informations/R MNCH/7Rastriya_Bal_Swaas

9. Kaur S, Randhawa RK. Effect of Biological Risk Factors and Home Environment on Motor Development in Early Child-Hood. International Journal of Medical Research \& Health Sciences, 2021, 10(1): 38-45

10. Olusanya BO, Davis AC, Wertlieb D, et al. Developmental disabilities among children younger than 5 years in 195 countries and territories, 1990-2016: a systematic analysis for the global burden of disease study 2016. Lancet Glob Health. 2018;6: e1100e1121.

11. Datar A, Jacknowitz A. Birth Weight Effects on Children's Mental, Motor, and Physical Development: Evidence from Twins Data. Matern Child Heal J. 2009;13: 780-794.

12. Whitaker AH, Feldman JF, Lorenz JM, et al. Motor and cognitive outcomes in nondisabled low-birth-weight adolescents: early determinants. Arch Pediatr Adolesc Med. 2006;160(10):1040-6.

13. Tavasoli A, Aliabadi F, Eftekhari R. Motor developmental status of moderately low birth weight preterm infants. Iran. J. Pediatr. 2014; 24:581-586.

14. Drozd-Dąbrowska M, Trusewicz R, Ganczak M. Selected Risk Factors of Developmental Delay in Polish Infants: A Case-Control Study. Int J Environ Res Public Health. 2018;15(12):2715.

15. Pirhadi M, Mohebbi DZ, Torabi F. The Relationship between Small for Gestational Age (SGA) at Birth and Developmental Delay in Children Aged 4 to 60 Months. Int J Pediatr 2018; 6(11): 8595-8603. 
16. Kerstjens JM, de Winter AF, Bocca-Tjeertes IF, et al. Risk of developmental delay increases exponentially as gestational age of preterm infants decreases: a cohort study at age 4 years. Dev Med Child Neurol 2012;54(12):1096-1101.

17. Schonhaut L, Armijo I, Pérez M. Gestational age and developmental risk in moderately and late preterm and early term infants. Pediatrics. 2015;135: e835-e841.

18. Hochstedler KA et al. Gestational Age at Birth and Risk of Developmental Delay: The Upstate KIDS Study.Am J Perinatol.2020 Mar 6;10.1055/s-00401702937.

19. Soleimani F, Bajalan Z, Alavi Majd H, et al. Relationship Between Gender and Development Status in Children. Archives of Rehabilitation. 2018; 18(4):338-345.

20. Richter J, Janson H. A validation study of the Norwegian version of the Ages and
Stages Questionnaires. Acta Paediatr. 2007; 96(5): 748-52.

21. Simpson GA, Colpe L, Greenspan S. Measuring functional developmental delay in infants and young children: prevalence rates from the NHIS-D. Paediatr Perinat Epidemiol. 2003;17(1):68-80.

22. Berglund E, Eriksson M, Westerlund M. Communicative skills in relation to gender, birth order, childcare and socioeconomic status in 18-month-old children. Scand J Psychol. 2005;46(6):485-91.

How to cite this article: Kaur S, Sidhu NS, Randhawa RK. Biological risk factors and early developmental delay assessment in infants using ages and stages questionnaire, version 3 (ASQ3). International Journal of Research and Review. 2021; 8(7): 272-277. DOI: https://doi. org/10.52403/ijrr.20210738 